LA-UR 97-4706

Approved for public release;

distribution is unlimited

\title{
Two-lane traffic rules for cellular automata: A systematic approach
}

Kai Nagel, Dietrich E Wolf, Peter Wagner, Patrice Simon

\section{LOS ALAMOS}

NATIONAL LABORATORY

Los Alamos National Laboratory, an affirmative action/equal opportunity employer, is operated by the University of California for the U.S. Department of Energy under contract W-7405-ENG36. By acceptance of this article, the publisher recognizes that the U.S. Government retains a non-exclusive, royalty-free license to publish or reproduce the published form of this contribution, or to allow others to do so, for U.S. Government purposes. Los Alamos National Laboratory requests that the publisher identify this article as work performed under the auspices of the U.S. Department of Energy. The Los Alamos National Laboratory strongly supports academic freedom and a researcher's right to publish; as an institution, however, the Laboratory does not endorse the viewpoint of a publication or guarantee its technical correctness. 



\title{
Two-lane traffic rules for cellular automata: A systematic approach
}

\author{
Kai Nagel,${ }^{a, *}$ Dietrich E. Wolf, ${ }^{b, \dagger}$ Peter Wagner,${ }^{c, d,{ }^{\ddagger}}$ and Patrice Simon ${ }^{a, \S}$ \\ ${ }^{a}$ Los Alamos National Laboratory, TSA-DO/SA Mail Stop M997, \\ Los Alamos NM 87545, U.S.A. \\ $b$ Theoretische Physik, Gerhard-Mercator-Universität, \\ 47048 Duisburg, Germany \\ ${ }^{c}$ Zentrum für Paralleles Rechnen (ZPR), Universität zu Köln, \\ 50923 Köln, Germany \\ ${ }^{d}$ Deutsches Zentrum für Luft- und Raumfahrt (DLR), Linder Höhe, \\ 51170 Köln, Germany
}

DRAFT DRAFT February 1, 2008 DRAFT DRAFT

February 1, 2008

\begin{abstract}
Microscopic modeling of multi-lane traffic is usually done by applying heuristic lane changing rules, and often with unsatisfying results. Recently, a cellular automaton model for two-lane traffic was able to overcome some of these problems and to produce a correct density inversion at densities somewhat below the maximum flow density. In this paper, we summarize different approaches to lane changing and their results, and propose a general scheme, according to which realistic lane changing rules can be developed. We test this scheme by applying it to several different lane changing rules, which, in spite of their differences, generate similar and realistic results. We thus conclude that, for producing realistic results, the logical structure of the lane changing rules, as proposed here, is at least as important as the microscopic details of the rules.
\end{abstract}

*kai@lanl.gov

${ }^{\dagger}$ d.wolf@uni-duisburg.de

$\ddagger$ p.wagner@zpr.uni-koeln.de

§simonp@tsasa.lanl.gov 
Figure 1: Traffic measurements in reality. Left column: as function of flow; right column: as function of density. Top: flow; middle: velocity; bottom: lane usage. The units for density are vehicles per kilometer per 2 lanes, and for flow they are vehicles per hour per 2 lanes. Each data point corresponds to a 1 minute average. Figure from Wiedemann, see [3] for further information.

\section{Introduction}

Much progress has been made in understanding single lane traffic by using simple models (e.g. 11, 2]). Although one could claim that these models also explain homogeneous multi-lane traffic, they definitely fail when traffic on different lanes behaves differently. If one wants to investigate lane specific dynamics, one has to address the question of how vehicles change from one lane to the other. Here we propose an elementary scheme to develop such rules and compare the simulation results of different realizations of this scheme with empirical data from the German highway.

The preferred approach in science is to start from first principles and then, using mathematics or simulation, to derive macroscopic relationships. In sciences which involve human beings this is hopeless: the gap between first principles and human behavior is too big. One alternative is to search heuristically for microscopically minimal "plausible" models which generate observed behavior on the macroscopic level. It is this approach that has often been used successfully when physics methods have been applied in the area of socio-economic systems. In this paper we want to go one step beyond that and look for systematic logical structures in the rule sets for lane changing.

Accordingly, we start out from real world data (Sec. 2), followed by a short review of traditional approaches to this problem in traffic science (Sec. 3). Sec. 4 outlines our approach. In the following three sections (Sec. $5-7$ ), we describe simulation results with different rules. Sec. 8 looks closer into the mechanism at flow breakdown near maximum flow in the two-lane models. Sec. 9 is a discussion of our work, followed by a section showing how other multi-lane models for cellular automata fit into our scheme (Sec. 10). The paper concludes with a short summary.

\section{Real world measurements}

As stated above, we are interested in macroscopic observations of traffic flow quantities related to lane changing behavior. A typical such measurement can look like Fig. 1. It contains measurements of density (in vehicles $/ \mathrm{km} / 2$ lanes), flow (in veh/h/2 lanes), velocity (in $\mathrm{km} / \mathrm{h}$ ) and lane usage (in \%), all averaged over one minute intervals. The left column shows velocity and lane usage as functions of flow; the right column shows flow, velocity, and lane usage as functions of density. For theoretical purposes, using flow as the control parameter has the disadvantage that for the same flow value one has two different 
regimes - at high density and at low density. For example in the lane usage plot, one cannot distinguish which data points belong to which regime. We will therefore concentrate on plots where density is the control parameter.

The top right plot shows the typical flow-density diagram. Flow first increases nearly linearly with density, until it reaches a maximum at $\rho \approx 40$ vehicles $/ \mathrm{km} / 2$ lanes and $q \approx 3500$ vehicles/hour $/ 2$ lanes. From there, flow decreases with increasing density, and the scatter of the values is much larger than be-

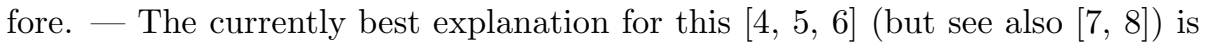
that, for low densities, traffic is roughly laminar and jams are short-lived. In consequence, the addition of vehicles does not change the average velocity much and flow is a linear function of density: $q=\rho v$. For high densities, traffic is an irregular composition of jam waves, and laminar outflow traffic between jams. Here, data points are arbitrary averages over these regimes, leading to a much larger variability in the measurements.

The plot of the velocity vs. density confirms this: There is an abrupt drop in the average velocity at $\rho \approx 40 \mathrm{veh} / \mathrm{km} / 2$ lanes. Yet, velocity is also not constant at lower densities, leading indeed to a curvature of the flow-vs.-density curve below the value $\rho \approx 40 \mathrm{veh} / \mathrm{km} / 2$ lanes, which can be explained by the increasing influence of the slower vehicles in multi-lane traffic.

The lane usage shows a peculiarity which is particularly strong in Germany. As should be expected, at very low densities all traffic is on the right lane. T But with increasing density, eventually more than half of the traffic is on the left lane. Only at densities above the maximum flow point, this reverts to an equal distribution of densities between lanes.

Fig. 1] does not show the flows of the individual lanes. Ref. [9] contains such plots. They show that the pointed peak of the overall flow is caused by a pointed peak in the flow of the left lane; flow on the right lane remains constant over a large density range.

All this suggests the interpretation that the flow breakdown mechanism on German autobahns is complicated, with flow breaking down on the left lane first and thus not allowing the right lane to reach its possible full capacity [10].

\section{Traditional approaches}

Sparmann [9] discusses a lane changing implementation for the microscopic Wiedemann-model 111. Following Wiedemann's proposition, he distinguishes between the wish to change lanes and the decision to change lanes. For a lange change from right to left, these two parts are:

- Wish to change lanes if on any of the two lanes there is another vehicle ahead and obstructing.

- Decision to actually change lanes if there is enough space on the other lane.

\footnotetext{
${ }^{1}$ For countries such as Great Britain or Australia, left and right have to be interchanged.
} 
Conversely, for changing from left to right:

- Wish to change lanes if on both lanes there is nobody ahead and obstructing.

- Decision to actually change lanes if there is enough space on the other lane.

According to the philosophy of the Wiedemann-approach, "obstructing" is defined in terms of so-called psycho-physiological thresholds, which depend mostly on speed difference and distance, and allow three outcomes: no obstruction, light obstruction, severe obstruction. Gipps [12] reports a similar model.

The results are reported to be satisfying, yet unrealistic in at least one respect: The density inversion between right and left lane near maximum flow is not reproduced.

The Wiedemann-approach is a time-discrete formulation of a stochastic differential equation and therefore continuous in space. Some recent work in traffic has used a cellular automata approach, which is coarse-grained discrete both in time and space. Early lane changing rules in the context of cellular automata models for traffic flow are due to Cremer and co-workers [13, 14]. Following Sparmann, they implemented lane changes in the following way: Lanes are changed to the left

- if a slower vehicle is less than $l_{l}$ cells ahead,

- and if a gap of size $\Delta x$ exists on the left lane;

lanes are changed to the right

- if, on the right lane, there is no slower vehicle less than $l_{r}$ cells ahead,

- and there is a gap of size $\Delta x$ on the right lane.

Again, they failed to reproduce the density inversion in the lane usage.

\section{Our approach}

Which contribution can Statistical Physics make in such a situation? The strength of Statistical Physics is to explain how microscopic relationships generate macroscopic behavior. Thus, the contribution of Statistical Physics in traffic science (or in socio-economic systems in general) will be to investigate which microscopic rules contribute to certain aspects of macroscopic behavior and how.

Since current psychological knowledge does not allow to define beyond doubt the set of microscopic rules involved in lane changing, we propose to construct these rules according to certain symmetries inherent in the problem. As we will point out, these symmetries simplify considerably the construction of consistent lane changing rules. 
Now, in spite of the absence of "first principles", it certainly still makes sense to have a "plausible" starting point. We thus state here what we will use as the elementary laws, and later, how we derive algorithmic rules from them. Similar to Ref. [9], we propose that the basic ingredients are security, legal constraints, and travel time minimization. Security requires to leave enough space between all vehicles. The legal constraints depend on the country. Travel time minimization means that one chooses the optimal lane under these constraints.

Let us start with security. Security means that one leaves enough space in front of and behind oneself. As long as one stays on one lane, this is ensured by single-lane driving rules, as e.g. given by the rules in Refs. [15, 16]. In the context of changing a lane this means that there must be enough space on the target lane. Technically, one can say that there must be a gap of size $g a p_{-}+1+g a p_{+}$. The label $+(-)$ belongs to the gap on the target lane in front of (behind) the vehicle that wants to change lanes. In the following we characterize the security criterion by the boundaries $\left[-g a p_{-}, g a p_{+}\right]$of the required gap on the target lane relative to the current position of the vehicle considered for changing lanes.

Different choices for both parameters are possible. Throughout this paper we use $g a p_{+}=v$ and $g a p_{-}=v_{\max }$ (i.e. $\left[-v_{\max }, v\right]$ ), where $v$ is the speed of the vehicle which changes lanes and $v_{\max }$ is the maximum velocity allowed in the cellular automaton.

Let us now go to legal constraints. For example in Germany, lane usage is regulated essentially by two laws: 1 . The right lane has to be used by default, and 2. passing has to be on the left. In the United States, the second law is considerably relaxed. In this paper, we will use "Germany" and "United States" as placeholders for two somewhat extreme cases. We expect that the behavior of many other countries will be found somewhere in between.

Travel time optimization means that lane changes to the left are triggered by a slow vehicle in the same lane ahead and when the target lane is more attractive (because of optimization). In this context, "slow" means a velocity smaller or equal to the one of the car behind. Here we give two examples, first for changing to left:

(a) German criterion. In Germany passing is not allowed on the right. Hence, if there is a slow vehicle on the left lane, one has to change to the left, behind that slow vehicle. Thus one changes to the left if there is a slow car ahead on the same lane or on the left:

$$
v_{r} \leq v . O R . v_{l} \leq v
$$

$v_{r}, v_{l}$ are taken within a certain distance one looks ahead, $d$, which is a free parameter. If there is no vehicle within this distance, the respective velocity is set to $\infty$.

(b) American criterion. By contrast, in America passing on the right is not explicitely forbidden. The left lane is only more attractive if the traffic there is faster than in one's own lane. Thus one changes from the right to 
the left if there is a slower car ahead in the same lane and if the next car in the left lane is faster than the car ahead:

$$
v_{r} \leq v . A N D . v_{r} \leq v_{l} .
$$

The easiest implementation of the law to use the right lane by default is to make the criterion for changing back to the right lane the logical negation of the criterion to change to the left lane; i.e. whenever the reason to change to the left lane ceases to exist, one changes back.

- This means for Germany that a change back to the right lane is tried as soon as the velocities of the cars ahead in both lanes are sufficiently large:

$$
v_{r}>v \cdot A N D . v_{l}>v .
$$

- In America, the rule would mean that one tries to change back if there is a faster car than oneself (or no car at all) in the right lane, or if traffic in the right lane is running faster than on the left lane:

$$
v_{r}>v . O R . v_{r}>v_{l} .
$$

In summary, a lane is changed if two criteria are fulfilled:

- - Security criterion: $\left[-v_{\max }, v\right]$ are fulfilled.

- - Incentive criterion: Is there a good reason to change lanes?

The examples above illustrate that the wish to change from right to left in general depends on both lanes. If the right lane is used by default, the criterion to change from left back to right is that the reason to change from right to left is no longer given, that is the negation of the former criterion.

However, if the right lane is not used by default, it is natural to consider symmetric incentive criteria: The return to the right lane then depends on the same criterion as the transition to the left lane, with "left" and "right" interchanged. The simplest example, which describes the actual American driving behavior fairly well, involves only one lane in contrast to our "American criterion" above: One changes lanes only when a slow vehicle is ahead:

- Criterion for change from right to left: $v_{r} \leq v$.

- Criterion for change from left to right: $v_{l} \leq v$.

This implies that vehicles stay on the left lane even when the right lane is completely empty, and describes that American drivers often do not use the rightmost lane in order to avoid the repeated disturbances due to slow vehicles coming from on-ramps. In the words of symmetric rules: When these drivers encounter one slow vehicle from on an on-ramp, they switch to the left lane and stay there until they run into a slower vehicle on that lane or until they want to get off the freeway. For that reason, TRANSIMS [17, 18] in its current 
microsimulation uses a totally symmetric lane-changing rule set. See Refs. [19, 20, 21] for symmetric lane changing rules.

Note that these considerations can easily be extended to multi-lane traffic. Also note that our paper only treats uni-directional traffic, i.e. all vehicles are headed into the same direction. Refs. [14, 22] are examples for the treatment of bi-directional traffic by cellular automata.

\section{Computer simulations of the basic velocity rules}

We now proceed to present computer simulations of the German rule-set to illustrate the above principles. Following Refs. 23, 20, 21], an update step of the whole system is divided into two major substeps: (i) lane changing, (ii) forward movement.

\subsection{Lane changing}

Lane changing here is implemented as a pure sideways movement. One should, though, better look at the overall result after the whole time step is completed, and then lane changing vehicles usually will have moved forwards, too. Still, the algorithm is underestimating the time vehicles usually need to change lanes: One CA iteration roughly corresponds to one second; lane changes in reality need about 3 sec $[9]$.

More specifically, the lane changing algorithm is an implementation of the following:

In even time steps, perform lane changes from right to left.2 All vehicles on the right lane for which the Incentive Criterion $\left(v_{r} \leq v . O R . v_{l} \leq v\right)$ and the Security Criterion $\left(\left[-v_{\max }, v\right]\right)$ are fulfilled are simultaneously moved to the left.

In odd time steps, perform lane changes from left to right. All vehicles on the left lane for which the Incentive Criterion $\left(v_{r}>v\right.$.AND. $\left.v_{l}>v\right)$ and the Security Criterion $\left(\left[-v_{\max }, v\right]\right)$ are fulfilled are simultaneously moved to the right.

The number of sites one looks ahead for the Incentive Criterion plays a critical role. Quite obviously, if one looks far ahead, one has a tendency to go to the left lane already far away from an obstructing vehicle, thus leading to a strong density inversion at low densities. Thus, this parameter can be used to adjust the density inversion. - The results described below were obtained with a lookahead of 16 sites, that is, if no vehicle was detected in that range on that lane, the corresponding velocity $v_{r}$ or $v_{l}$ was set to $\infty$.

\footnotetext{
${ }^{2}$ We separate changes from left to right and changes from right to left in anticipation of three lane traffic. In three lane traffic, in a simultaneous update it is possible that a vehicle from the left lane and a vehicle from the right lane want to go to the same cell in the middle lane. From a conceptual viewpoint of simulation, this may be called a scheduling conflict. Such conflicts can be resolved by, e.g., different update schedulings (such as here) 24, 25].
} 


\subsection{Forward movement}

The vehicle movement rules (ii) are taken as the single lane rules from Nagel and Schreckenberg [15, 16, which are by now fairly well understood 14, 26, 27.

For completeness, we mention the single lane rules here. They are

- IF $\left(v<v_{\max }\right)$ THEN $v:=v+1 \quad$ (accelerate if you can)

- IF ( $v>$ gap ) THEN $v:=$ gap (slow down if you must)

- IF $(v \geq 1)$ THEN WITH PROBABILITY $p$ DO $v:=v-1$ (sometimes be not as fast as you can for no reason).

These rules for forward movement will be used throughout the paper. , with $p$ equal to 0.25 . All simulations are performed in a circle of length $L=10000$. The maximum velocity is $v_{\max }=5$.

\subsection{Results}

As shown in Fig. 2, these rules generate reasonable relations between flow, density, and velocity. More importantly, they generate the density inversion below maximum flow which is a so important aspect of the dynamics on German freeways. Note that, maybe contrary to intuition, it is not necessary to have slow vehicles in the simulations in order to obtain the density inversion.

\section{Computer simulations of gap-rules}

For comparison, we also simulated a version of Wagner's "gap-rules" 28, 29], which is adapted to our classification scheme above. The reason to change to the left then becomes

$$
\operatorname{gap}_{r}<v_{\max } . O R . \operatorname{gap}_{l}<v_{\max },
$$

i.e. one has a reason to change to the left when there is not enough space ahead either on the right or on the left lane.

As stated above, as reason to change to the right we take the negation, although we allow for some "slack" $\Delta$ :

$$
\operatorname{gap}_{r} \geq v_{\max }+\Delta \cdot A N D . g a p_{l} \geq v_{\max }+\Delta,
$$

i.e. one changes from left to right if on both lanes there is enough space ahead.

The "slack" parameter $\Delta$ has been introduced in Ref. [28]. The larger it is the less inclined is the driver to change back to the right lane, and hence the more pronounced is the lane inversion. In this sense the parameter Delta plays a similar rôle in these gap-rules as the look-ahead distance in the basic velocity rules discussed before. We will use $\Delta=9$, the same value as in Ref. [29.

Fig. 3 shows results of simulations with these rules. One immediately notes that these rules both qualitatively and quantitatively generate the correct den-

sity inversion at maximum flow, i.e. at $\rho \approx 38 \mathrm{veh} / \mathrm{km} / 2$ lanes; but from there 

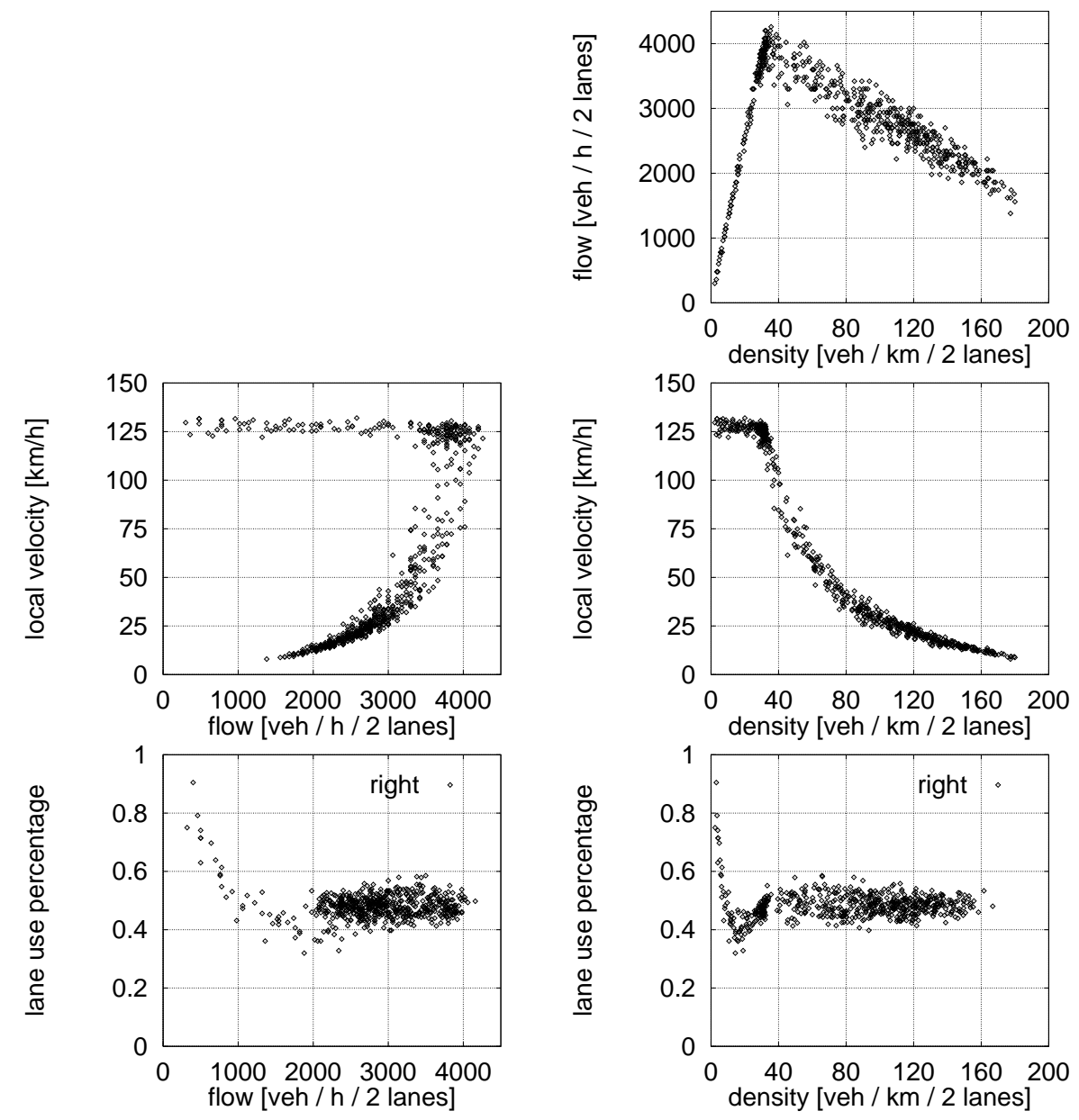

Figure 2: Simulation results for basic version of the velocity-based lane changing rules. Same type of plots as in Fig. 1. Each data point is a one minute average, except for lane usage, where each data point is a three minute average.

on with further increasing density the density inversion increases further, contrary to reality. Ref. 29] uses rules which (i) prohibit passing on the right and (ii) symmetrize traffic at very high densities; as a result, lane usage becomes much more symmetric above the density of maximum flow.

\section{Extensions for reality}

After having shown that both velocity-based and gap-based lane changing rules, based on the introduced logical scheme, can generate the density inversion effect, we now proceed to include more realism to bring the result closer to Wiede- 

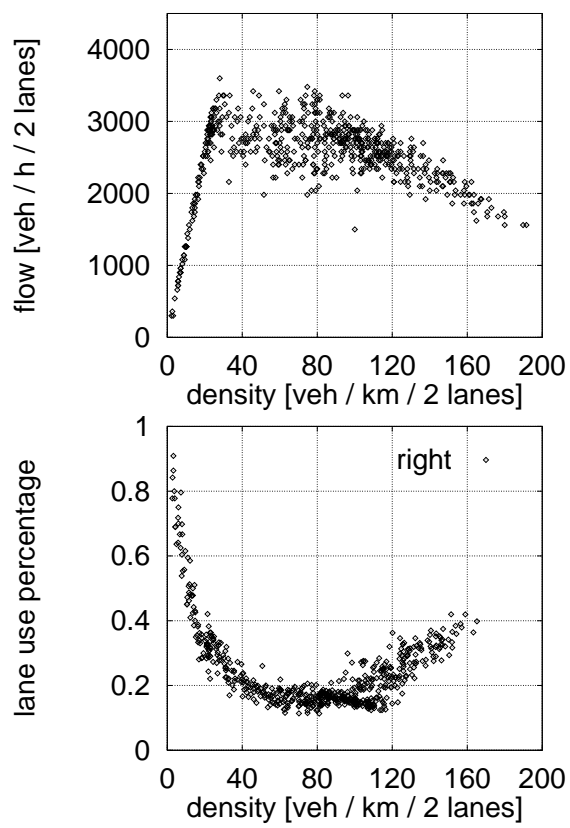

Figure 3: Simulation results for gap-based lane changing rules. Top: Flow vs. density. Bottom: Lane usage vs. density.

mann's data (Fig. 1).

\subsection{Slack}

With the basic velocity-based rules, one can adjust the density inversion to the correct lane use percentage, but the maximum inversion is reached at too low densities (at approx. $16 \mathrm{veh} / \mathrm{km} / 2$ lanes compared to approx. $28 \mathrm{veh} / \mathrm{km} / 2$ lanes in reality). One possibility to improve this is to introduce some slack $\Delta=3$ into the rules similar to the slack in the gap-based rules, i.e. vehicles change to the left according to the same rules as before, but the Incentive Criterion for changing back is not the inversion of this. Instead, it now reads

$$
v_{r}>v+\Delta . A N D . v_{l}>v+\Delta .
$$

Since these rules tend to produce a stronger density inversion than before, we reduced the look ahead value to 7 to obtain realistic lane usage values. Results are shown in Fig. 4 .

\subsection{Slack plus symmetry at high densities/low velocities}

In order to be able to tune the onset density as well as the amount of lane inversion the second parameter "slack" has been introduced in addition to the 

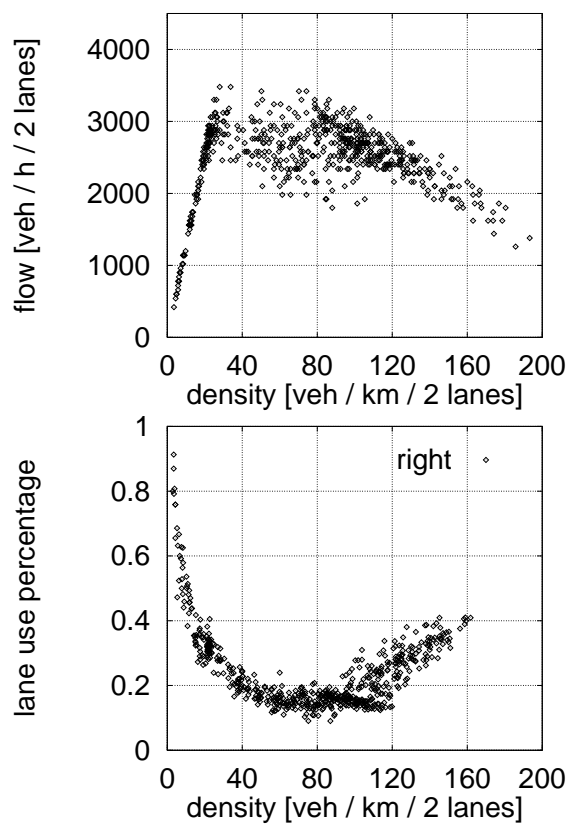

Figure 4: Simulation results for velocity-based lane changing rules with slack (i.e. there is some "slack" in the incentive criterion for changing to the right compared to the one for changing to the left). Top: Flow vs. density. Bottom: Lane usage vs. density.

look-ahead. This, however, has the side effect that traffic never reverts to an equal lane usage, even at very high densities, similar to what we obtained with the gap-rules above. In order to improve this, we make the rule-set symmetric at zero speed. In technical terms, this means that a vehicle at speed zero only checks if the speed on the other lane is higher than on its own lane, and if so, attempts to change lanes (restricted by the security criterion). Other solutions are possible to achieve this (see, e.g., Ref. [29]; or one could attempt to make the look-ahead distance velocity-dependent, e.g. $\propto v$ ). Fig. 目 shows that our approach indeed works, i.e. the lane usage at high densities now goes indeed to approximately $50 \%$ for each lane.

\subsection{Slow vehicles}

Wiedemann's data includes 10 percent trucks. We model the effect of trucks by giving 10 percent of the vehicles a lower maximum velocity 20, 23, 30]. Note that this only models the lower speed limit which is in effect for trucks in most European countries, but not the lower acceleration capabilities. The result for the flow-density curve and for the lane usage is shown in Fig. 6. The main difference to before is that the maximum flow is shifted towards higher 

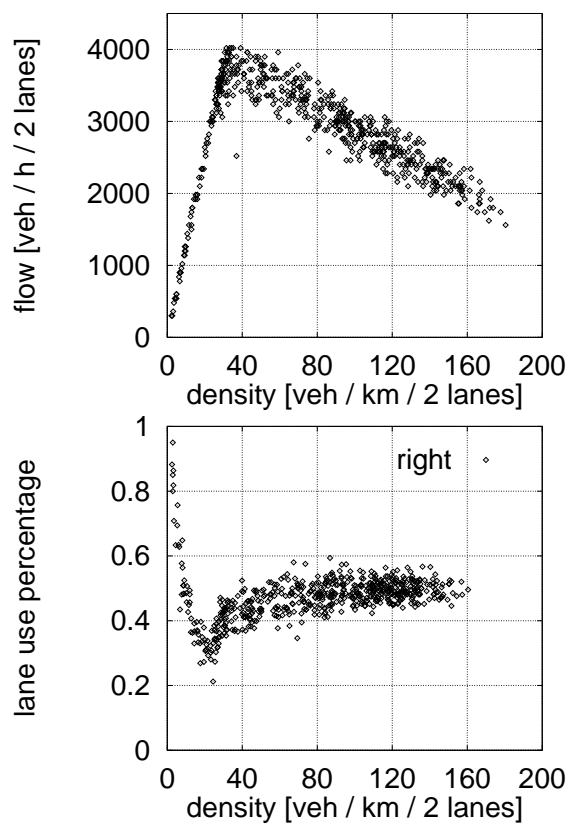

Figure 5: Plots when slack is used and symmetry at low velocities included. Top: Flow vs. density. Bottom: Lane usage vs. density.

densities, and there are more fluctuations in that region [20].

\subsection{Combination of all extensions}

Last, we show simulation results where all the above improvements (trucks; symmetry at high densities; slack) are used simultaneously (Fig. (7). Indeed, the results are now close to reality (cf. Fig. 11).

\section{The flow breakdown mechanism near maxi- mum flow}

One of the questions behind this research was to investigate if, in highly asymmetric two-lane systems, flow breakdown is indeed triggered by a single lane flow breakdown on the left lane. In order to address this question, we will, in the following, study space-time plots of the respective traffic dynamics as well as fundamental diagrams by lane. Since it turns out that traffic without slow vehicles is fundamentally different from traffic with slow vehicles, we will treat the two situations separately. 

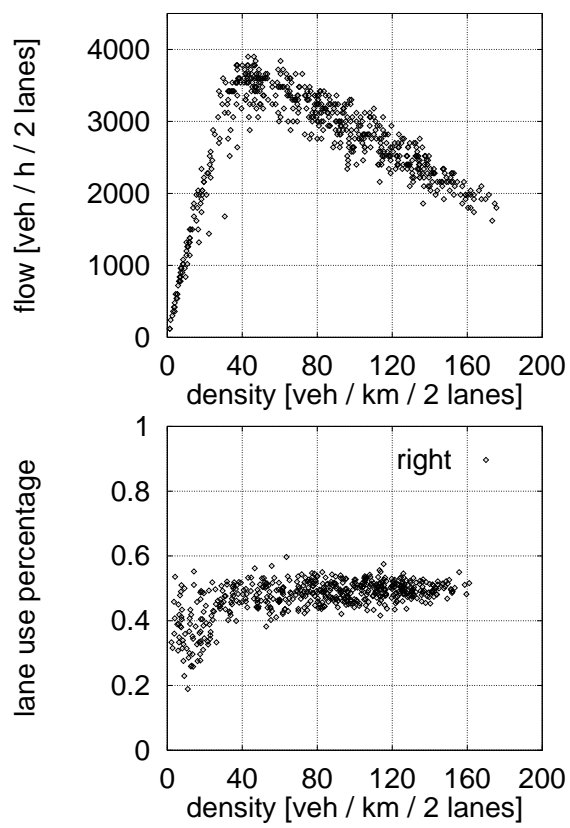

Figure 6: Plots when slow vehicles included. Top: Flow vs. density. Bottom: Lane usage vs. density.

\subsection{Maximum flow without slow vehicles}

Figs. 8 and 9 compare space-time plots from a one-lane situation with the twolane situation using the "basic" velocity-based lane changing rules, in both cases approximately at maximum flow. Not much difference in the dynamics is detectable except that maybe the 2-lane plot shows more small fluctuations instead of fully developed jams. This is confirmed by the single-lane fundamental diagrams for the systems (Fig. 10): The fundamental diagram for the left lane of basic velocity-based lane changing rules looks very similar to the corresponding 1-lane diagram, and also the right lane does not look much different. Also, the density inversion has reverted to 50:50 at maximum flow (Fig. 2).

Thus, the approach to maximum flow via increasing density is better described in the way that the left lane reaches maximum flow earlier than the right lane, and from then on all additional density is squeezed into the right lane. Only when the combined density of both lanes is above the maximum flow density, flow break-down happens. This argument gets confirmed by the observation that there are many measurement points near maximum flow in all fundamental diagrams, whereas at densities slightly higher than this significantly fewer data points exist. This should be compared to the situation which includes slower vehicles, which will be explained next. 

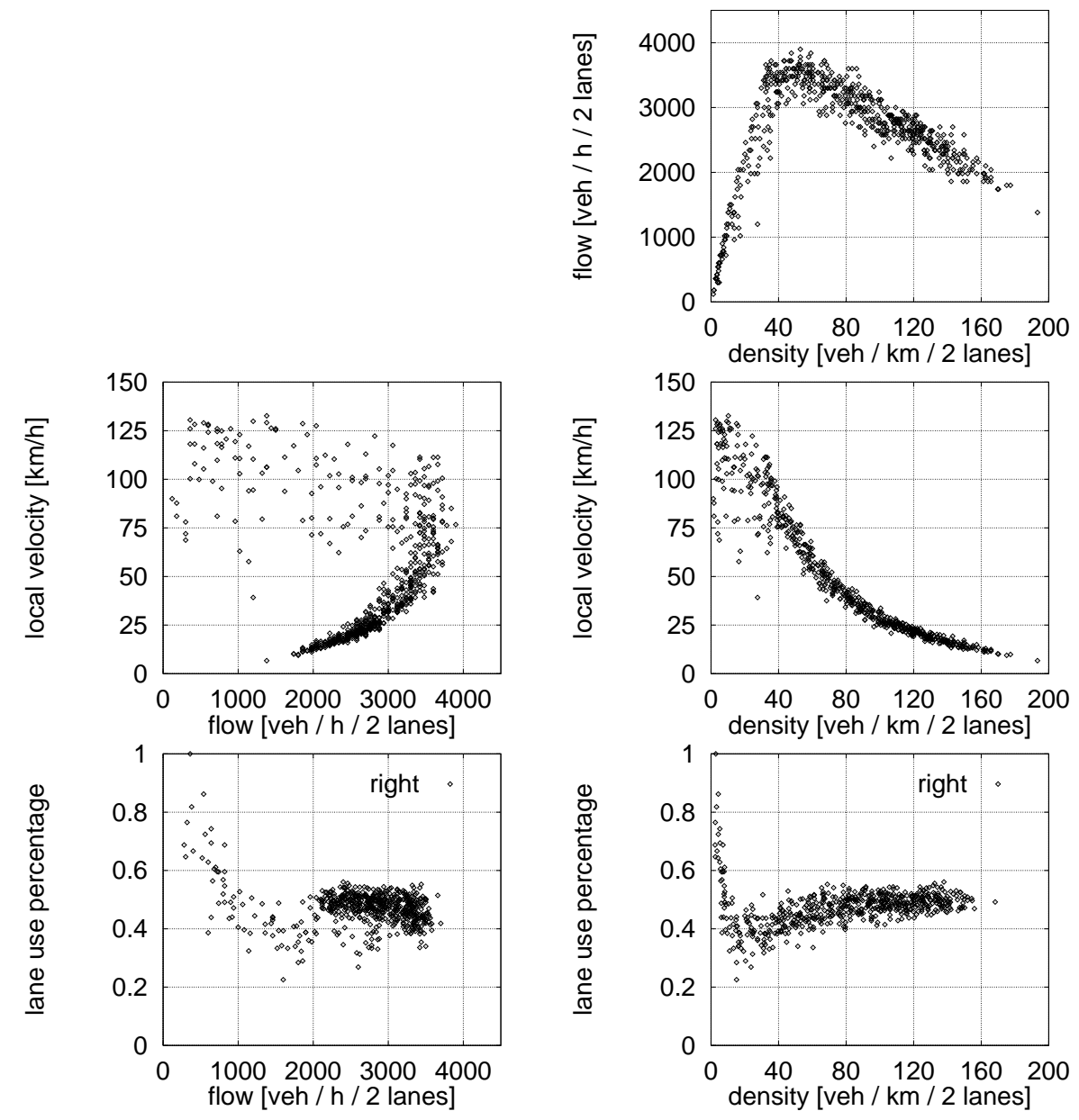

Figure 7: All three extensions of the basic rule-set (trucks; symmetry at high density; slack) are included. Compare this figure to Fig. 1.

\subsection{Maximum flow with slow vehicles}

The situation when slow vehicles are present is markedly different. The 2lane situation with slow vehicles (Fig. 12) looks more like the 1-lane situation with slow vehicles (Fig. 11) than like the 2-lane situation without slow vehicles (Fig. 9). That means: The presence of slow vehicles has a stronger influence on the dynamics than the difference between 1-lane and 2-lane traffic. The dominating feature is that fast vehicles jam up behind slow vehicles and get involved in start-stop dynamics which gets worse with increasing distance from the leading slow vehicle. In the 2-lane situation, these "plugs" are caused by two slow vehicles side by side; a situation which is empirically known to happen regularly. 


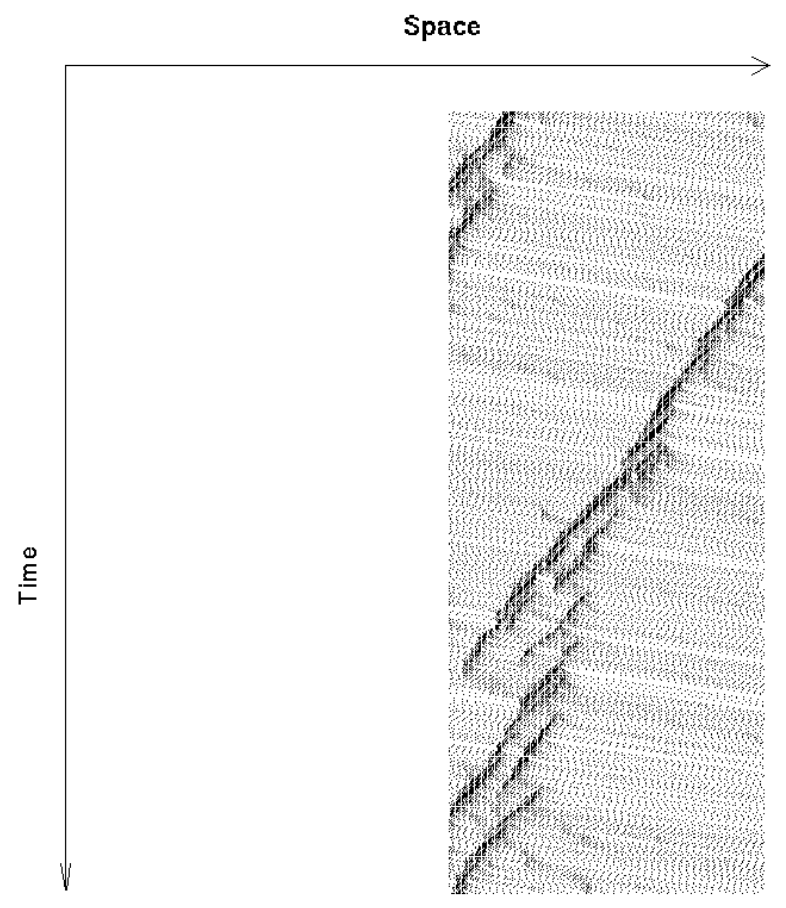

Figure 8: Space-time plot of one-lane traffic without slow vehicles.

For the "basic" lane changing rules, the queues behind the "plugs" have similar length on both lanes, both near the density of maximum flow (Fig. 12) and at lower densities (Fig. 13). In contrast, when using the lane changing rules with slack and symmetrization, then in the same situation, there are more vehicles behind the truck on the left than there are behind the truck on the right (Fig. 14). Experience seems to indicate that the more complicated rule-set is the more realistic one here.

The lane-based fundamental diagrams (Fig. 15) confirm the observation that slow vehicles change the dynamics. The marked peak and the accumulation of data points near maximum flow are both gone; maximum flow is found over a wider density range than before. The flow on the left lane generally reaches higher values both than flow on the right lane, and than single-lane traffic flow.

Space-time plots (Figs. 12 and 13) show why this is the case. Traffic in this situation is composed of two regimes:

- "Plugs" of slow vehicles side by side, and faster vehicles queued up behind them.

- "Free flow" regions, where the slow vehicles stay on the right and the fast vehicles are mostly on the left.

At low density, there are mostly free flow regions and a couple of "plugs" with 


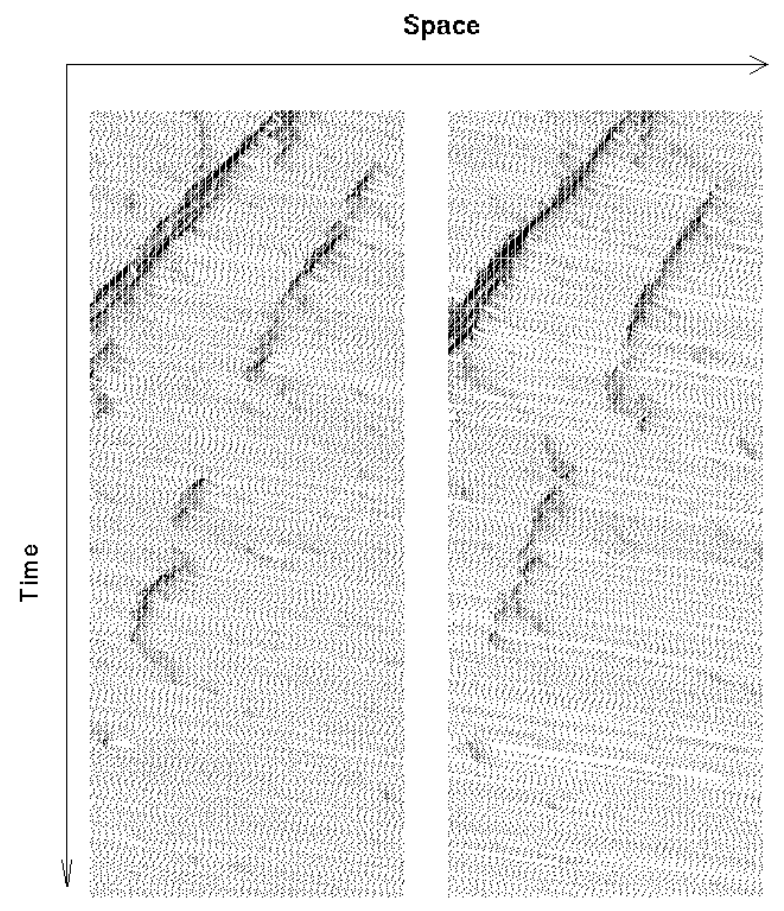

Figure 9: Space-time plot of two-lane traffic with the "basic" lane changing rules without slow vehicles. Left: left lane. Right: right lane.

queues behind them. With increasing density, the share of the free flow regions decreases while the share of the queueing regions increases. Eventually, the free flow regions get absorbed by the queueing regions, a 2-lane variant of the mechanism described in Ref. [31, 32.

From visual inspection, it is clear that up to that density (approx. $40 \mathrm{veh} / \mathrm{km} / 2$ lanes) the left lane carries a higher flow since it only has fast cars in the free flow regions. Above this density, it is clear that now also the slow vehicles get slowed down by the end of the queue ahead of them.

\section{Discussion}

(i) In spite of widespread efforts, many earlier models were not able to reproduce the lane inversion. Why is that so? The reason is that the lane inversion is a subtle spatial correlation effect: "I stay on the left if there is somebody ahead on the left." Indeed, some of the earlier models 14, 23] do not contain this crucial rule. Sparmann [9] contains it but still does not reproduce the density inversion; so one would speculate that the weight for this rule was not high enough.

(ii) Real-world traffic seems to be more stable in the laminar regime than 

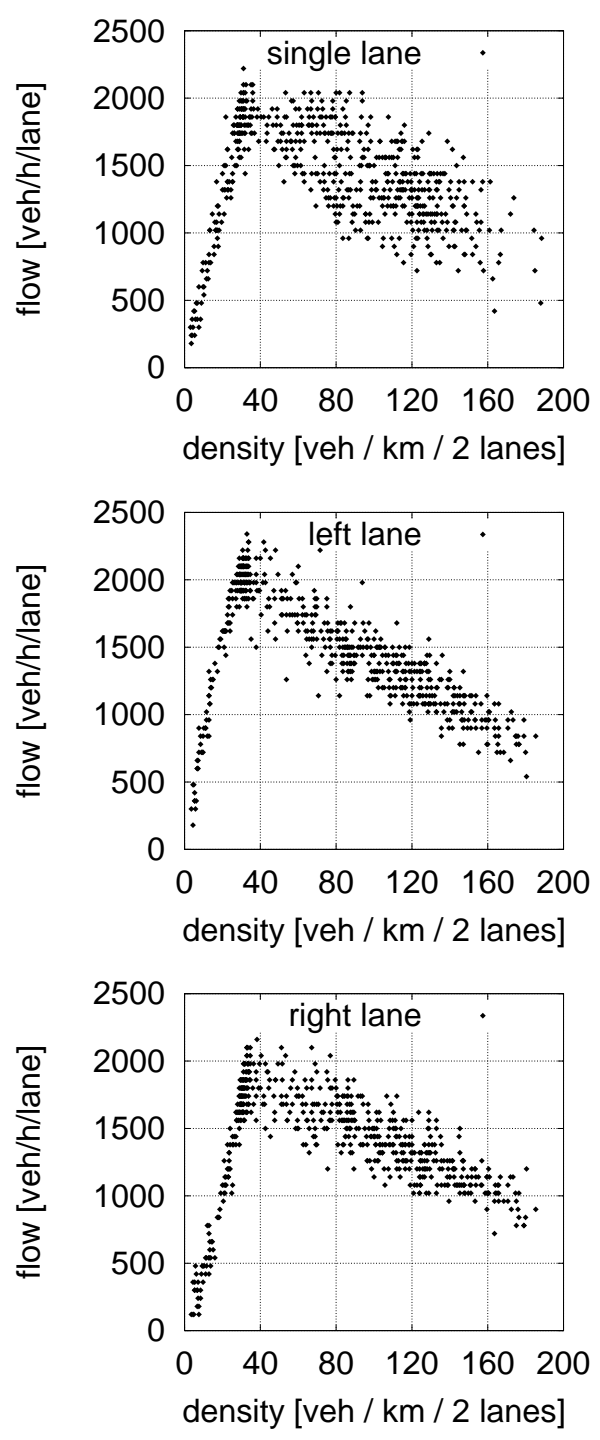

Figure 10: (a) Fundamental diagram for single-lane rules. (b) Fundamental diagram for left lane of basic velocity two-lane rules, i.e. plotting flow on the left lane vs. density on both lanes for 1-minute averages. (c) Fundamental diagram for right lane of basic velocity two-lane rules.

our simulated two-lane traffic. This can be seen in the "overshoot" (hysteresis, see Ref. [33) of the low-density branch of the flow-density-plot which is more pronounced in reality than in the results of this paper. The single-lane model [34] looked more realistic here. Yet, recent research shows that the hysteresis effect is 


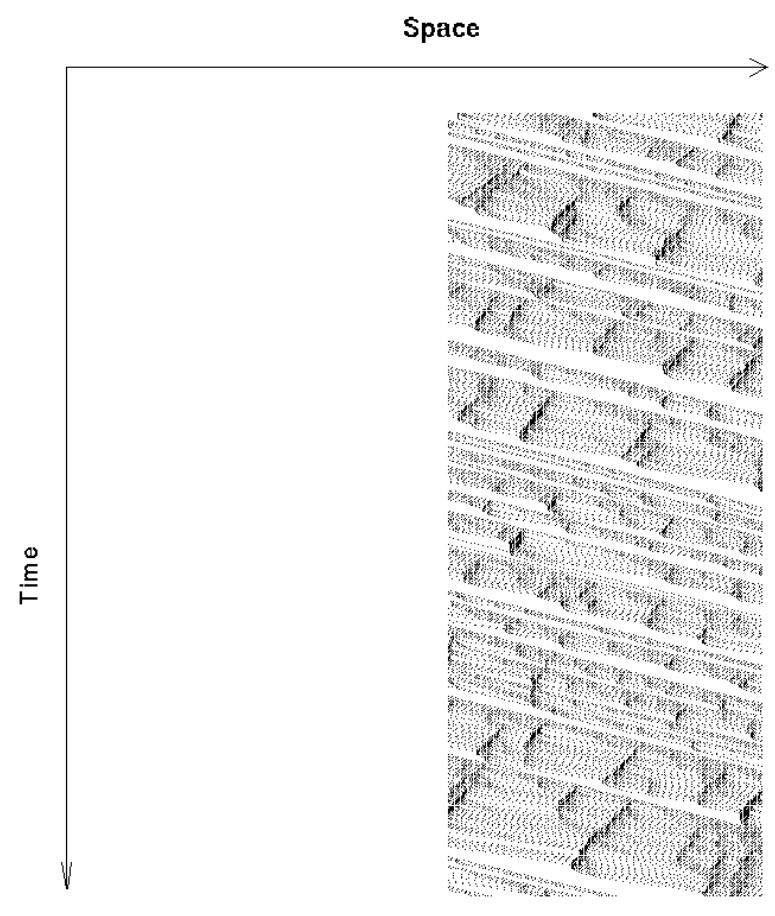

Figure 11: Space-time plot of one-lane traffic near maximum flow including $10 \%$ slow vehicles.

actually related to the structure of the braking rules of the single-lane velocity rules [6, 35. More precisely: In models with more refined braking rules the laminar traffic does not break down that easily because small disturbances can be handled by small velocity adjustments.

In this context, it should be stressed that, as mentioned above, our plots actually show three minute averages for the lane usage plots whereas all other plots are generated from one minute averages. The reason for this is that one minute averages for lane usage had so much variance that the overall structure was not visible. Yet, in reality one minute averages are sufficient also for this quantity. This indicates that our models have, for a given two-lane density, a higher variation in the lane usage than reality has. - Also, the plots of velocity vs. flow indicate that the range of possible velocities for a given flow is wider in the simulations than in reality, again indicating that for a given regime, our model accepts a wider range of dynamic solutions than reality.

(iii) The fact that we needed space-time plots for resolving many of the dynamical questions indicates that the methodology of plotting short time averages for density, flow, and velocity, has shortcomings. The reason has been

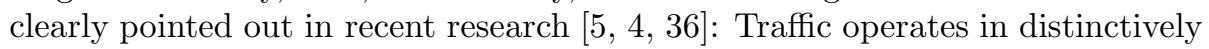
different dynamic regimes, two of them being laminar traffic and jammed traffic. 


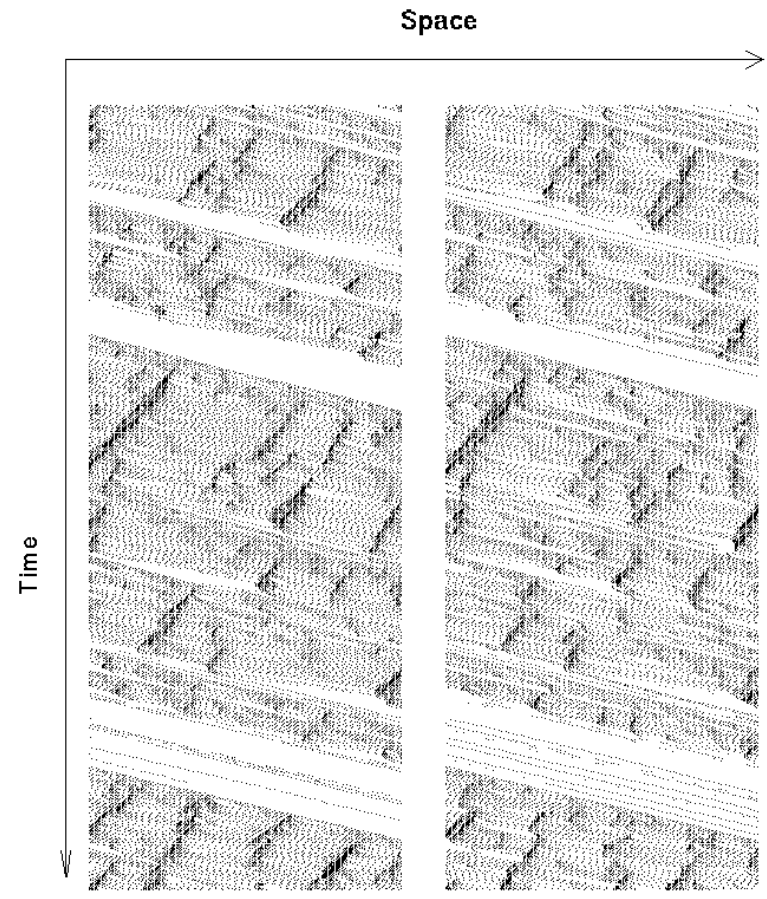

Figure 12: Space-time plot of two-lane traffic near maximum flow including $10 \%$ slow vehicles using the "basic" velocity-based lane changing rules of this paper. Left: left lane. Right: right lane.

Averaging across time means that often this average will, say, contain some dynamics from the laminar regime and some dynamics from the jammed regime, thus leading to a data point at some intermediate density and flow.

In transportation science, it seems that this problem is empirically known because people are using shorter and shorter time averages (1-min averages instead of 5-min averages used a couple of years ago or 15-min averages used ten or more years ago). It seems that one should try vehicle based quantities. Plotting $v / \Delta x$ as a function of $1 / \Delta x$, where $\Delta x$ is the front-bumper to front-bumper distance between two vehicles, is still a flow-density plot, but now individualized for vehicles. Instead of just plotting data point clouds, one would now have to plot the full distribution (i.e. displaying the number of "hits" for each flow-density value).

\section{Other two-lane models}

It is possible to review earlier lane changing models in the view of the scheme presented in this paper. In general, classifying some of the earlier rules into our 


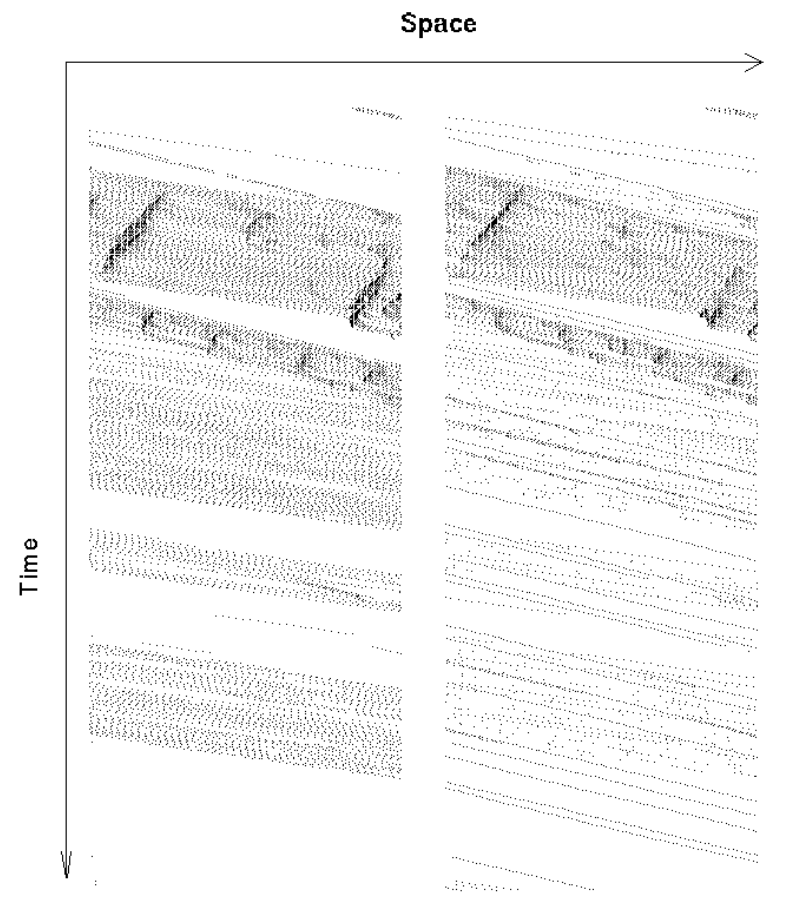

Figure 13: Space-time plot of two-lane traffic at about half the density of maximum flow, including $10 \%$ slow vehicles, using the "basic" lane changing rules of this paper. Same as Fig. 12, except for the lower density. Left: left lane. Right: right lane.

scheme is sometimes difficult, but usually possible. For example, when one uses

$$
\operatorname{gap}_{r}<v_{\max } . \text { OR. } \text { gap }_{l}<v_{\max }
$$

as a reason to change to the left, then the negation of that, including "slack" $\Delta$, would be the reason to change to the right. Let us also use a security criterion as follows:

$$
\text { gap }-=v_{b a c k}+1
$$

(i.e. the distance to the car behind on the other lane should be larger than its velocity) and

$$
g a p_{+}=\min \left[g a p+1, v_{\max }\right]
$$

(i.e. the distance to the car ahead on the target lane should be larger than either (i) the distance to the car ahead on the current lane, or (ii) the maximum velocity). With the exception of the addition of the second part of the Incentive Criterion to change left, these are exactly the same rules as used in Ref. 29].

Note, though, that this is not completely trivial. For example, the incentive to change left "gap $>$ gap " of Ref. [29] is now in the security criterion. Also, 


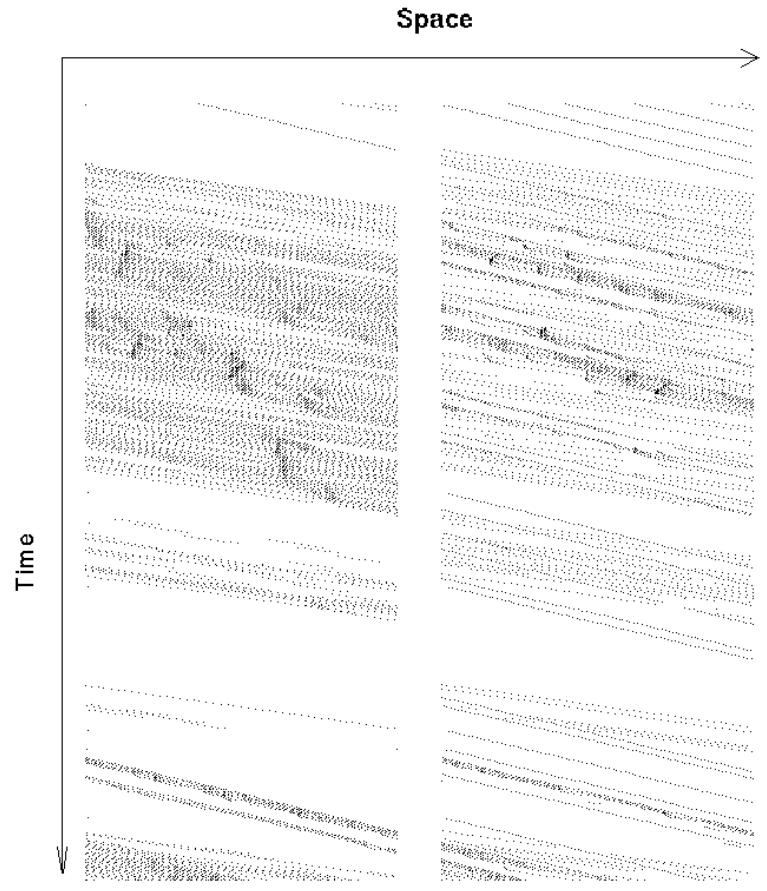

Figure 14: Space-time plot of two-lane traffic at about half the density of maximum flow, including $10 \%$ slow vehicles, using the lane changing rules with slack and symmetrization. Left: left lane. Right: right lane. Trajectories of fast vehicles (less steep slope) on the right lane which seem to go "through" the slow vehicles (steep slope) are actually interrupted and go to the left lane for short times.

for changes from left to right, the forward part of the security criterion could be left out, at least for the values of $\Delta$ which have been used. Quite generally, it can happen that a rule can fit into our logical scheme, but parts of the decision tree can never be reached so that parts of the rule can be omitted without changing anything.

Indeed, many asymmetric lane changing rules investigated in the literature can be viewed through our characterization. Table 1 contains many asymmetric lane changing rules from the traffic cellular automaton literature. The underlined parts have been added to make the rules completely fit into our scheme, i.e. to make the incentive to change to the right the logical negation (sometimes including "slack") of the incentive to change to the left. It would be interesting to test if the neglected part of the rules would be used often or not if they were actually implemented. 

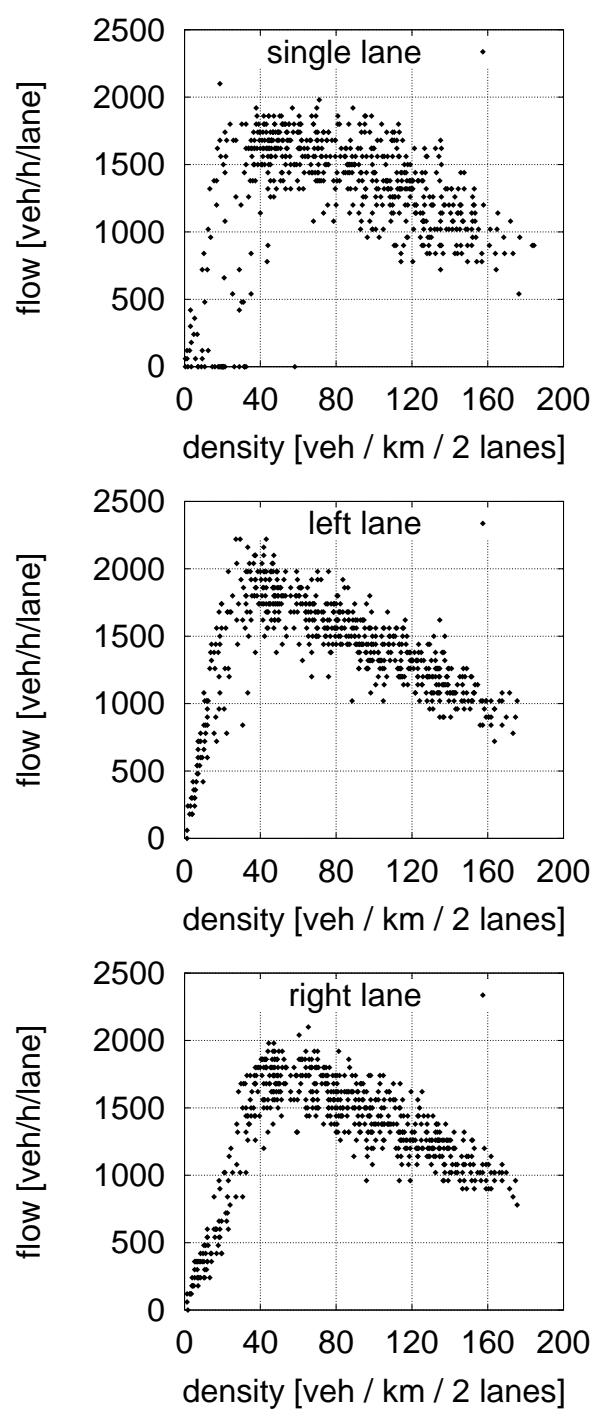

Figure 15: Simulation results for traffic including 10\% slow vehicles: (a) Fundamental diagram for single-lane rules. (b) Fundamental diagram for left lane of basic velocity-based two-lane rules, i.e. plotting flow on the left lane vs. density on the left lane for 1-minute averages. (c) Fundamental diagram for right lane of basic velocity two-lane rules.

\section{Summary}

This paper classifies the multitude of possible lane changing rules for freeway traffic. The first part of this follows Sparmann [9]: One can separate the rules 
into an "incentive to change lanes" and a security criterion, which asks if there is enough space available on the target lane.

The second part of this is the observation that in countries with a default lane and a passing lane, the incentive to change right is just the logical negation of the incentive to change left, with possibly some slack (inertia).

The security criterion seems to be universal for all reasonable lane changing rules: $\left[-g a p_{-}, g a p_{+}\right]$has to be empty on the target lane; the exact values of the parameters $g a p_{-}$and $g a p_{+}$do not seem to matter too much as long as they are reasonably large. We used $g a p_{-}=v_{\max }$ and $g a p_{+}=v$.

For the Incentive Criterion we argue that its general structure for highly asymmetric traffic has to be "change to the left when either on your lane or on the left lane somebody is obstructing you", and "change back when this is no longer true". Since this usually leads to a generic density inversion at high densities, one has to add a symmetrizing rule for high density traffic. We simply used a symmetric Incentive Criterion for vehicles with velocity zero.

Both velocity and gap based implementations of this give satisfying results.

Further, we showed that most asymmetric lane changing models in the physics literature fit into this scheme.

\section{Acknowledgements}

We thank R. Wiedemann for making Fig. I available to us. This work has been performed in part under the auspices of the U.S. Department of Energy at Los Alamos National Laboratory, operated by the University of California for the U.S. Department of Energy under contract W-7405-ENG-36, and at the HLRZ, Forschungszentrum Jülich. DW and KN also thank the Deutsche Forschungsgemeinschaft for support.

\section{A Transformation of the Wagner's rules from Ref. [28]}

Finding a correspondance for the rules of Wagner in Ref. [28] is not straightforward. However, at closer inspection, the rules turn out to be inconsistent for certain choices of parameters. The forward part of the Incentive Criterion is:

$$
\begin{aligned}
& R \rightarrow L: \operatorname{gap}_{r}<v_{\max } . A N D . g_{a p_{l}}>g a p_{r} \\
& L \rightarrow R: \operatorname{gap}_{r}>v+\Delta^{\prime} . A N D \cdot g_{a p_{l}}>v+\Delta^{\prime}
\end{aligned}
$$

Assume for example a case where $\operatorname{gap}_{r}=3, \operatorname{gap}_{l}=4, v=0, v_{\max } \geq 4$, and $\Delta^{\prime}=0$. Then the vehicle does not want to be in either lane.

This problem gets resolved for $\Delta^{\prime} \geq v_{\max }-1$; and indeed $\Delta^{\prime} \geq 6$ was used.

Now, if one assumes $\Delta^{\prime} \geq v_{\max }-1$, then one can simplify the rule-set. One can move the condition $g a p_{l}>g a p_{r}$ into the security criterion $g a p_{+} \geq$ 
$\min \left[\operatorname{gap}+1, v_{\max }\right]$, and the remaining incentives to change lanes are:

$$
\begin{aligned}
& R \rightarrow L: \operatorname{gap}_{r}<v_{\max } \underline{. O R . g a p_{l}<v_{\max }} \\
& L \rightarrow R: \operatorname{gap}_{r} \geq v_{\max }+\Delta(v) . A N D . g p_{l} \geq v_{\max }+\Delta(v),
\end{aligned}
$$

where, as in Table 1, the underlined part is added to make the rule fit into the scheme. Note that in this interpretation, the slack now is $\Delta(v)=\Delta^{\prime}-v_{\max }+v$, i.e. a function of the velocity.

\section{References}

[1] D.E.Wolf, M.Schreckenberg, and A.Bachem, editors. Traffic and granular flow. World Scientific, Singapore, 1996.

[2] D.E. Wolf and M. Schreckenberg, editors. Traffic and granular flow II. Springer, in press.

[3] R. Wiedemann. Beschreibung des Staus. In H. Keller, editor, Beiträge zur Theorie des Straßenverkehrs. Forschungsgesellschaft für Straßen- und Verkehrswesen, Köln, Germany, 1995.

[4] K. Nagel. Particle hopping models and traffic flow theory. Phys. Rev. E, 53(5):4655, 1996.

[5] B.S. Kerner and P. Konhäuser. Structure and parameters of clusters in traffic flow. Physical Review E, 50(1):54, 1994.

[6] S. Krauss, P. Wagner, and C. Gawron. Metastable states in a microscopic model of traffic. Physical Review E, 55(5):5597-5602, 1997.

[7] B.S. Kerner and H. Rehborn. Experimental properties of complexity in traffic flow. Physical Review E, 53(5):R4275-R4278, 1996.

[8] B.S. Kerner and H. Rehborn. Experimental properties of phase transitions in traffic flow. Physical Review Letters, 79(20):4030-4033, 1997.

[9] U. Sparmann. Spurwechselvorgänge auf zweispurigen BABRichtungsfahrbahnen. Number 263 in Forschung Straßenbau und Straßenverkehrstechnik. Bundesminister für Verkehr, Bonn-Bad Godesberg, Germany, 1978.

[10] W. Brilon, personal communication.

[11] R. Wiedemann. Simulation des Straßenverkehrsflusses. Schriftenreihe Heft 8, Institute for Transportation Science, University of Karlsruhe, Karlsruhe, Germany, 1994.

[12] P. G. Gipps. A model for the structure of lane-changing decisions. Transportation Research B, 20B(5):403-414, 1986. 
[13] M. Cremer and J. Ludwig. A fast simulation model for traffic flow on the basis of Boolean operations. Mathematics and Computers in Simulation, 28:297-303, 1986.

[14] H. Schütt. Entwicklung und Erprobung eines sehr schnellen, bitorientierten Verkehrssimulationssystems für Straßennetze. Schriftenreihe Heft 6, AG Automatisierungstechnik TU Hamburg-Harburg, Hamburg-Harburg, Germany, 1991.

[15] K. Nagel. Freeway traffic, cellular automata, and some (self-organizing) criticality. In R.A. de Groot and J. Nadrchal, editors, Physics Computing '92, page 419. World Scientific, 1993.

[16] K. Nagel and M. Schreckenberg. A cellular automaton model for freeway traffic. J. Phys. I France, 2:2221, 1992.

[17] TRANSIMS, TRansportation ANalysis and SIMulation System, Los Alamos National Laboratory, Los Alamos, U.S.A. See www-transims.tsasa.lanl.gov.

[18] K. Nagel, P. Stretz, M. Pieck, S. Leckey, R. Donnelly, and C.L. Barrett. TRANSIMS traffic flow characteristics, TRB preprint 981332. Also Los Alamos Unclassified Report LA-UR 97-3530 (see www-transims.tsasa.lanl.gov/research_team/).

[19] T. Nagatani. Dynamical jamming transition induced by a car accident in traffic-flow model of a two-lane roadway. Physica A, 202:449-458, 1994.

[20] M. Rickert. Simulationen zweispurigen Autobahnverkehrs mit Zellularautomaten. Master's thesis, University of Cologne, Köln, Germany, 1994.

[21] M. Rickert, K. Nagel, M. Schreckenberg, and A. Latour. Two lane traffic simulations using cellular automata. Physica A, 231:534, 1996.

[22] P.M. Simon and H.A. Gutowitz. Cellular automaton model for bidirectional traffic. Physical Review E, 57(1):in press, 1998.

[23] A. Latour. Simulation von Zellularautomaten-Modellen für Mehrspurverkehr. Master's thesis, University of Cologne, 1993.

[24] C.L. Barrett. Personal communication.

[25] Barrett C.L. and Reidys C.M. Elements of a theory of simulation I: sequential CA over random graphs. Appl. Math. and Comp., to appear.

[26] M. Sasvari and J. Kertesz. Cellular automata models of single lane traffic. Physical Review E, 56(4):4104-4110, 1997.

[27] B. Eisenblätter, L. Santen, A. Schadschneider, and M. Schreckenberg. Jamming transition in a cellular automaton model for traffic flow. Physical Review E, in press. 
[28] P. Wagner. Traffic simulations using cellular automata: Comparison with reality. In D E Wolf, M.Schreckenberg, and A.Bachem, editors, Traffic and Granular Flow, Singapore, 1996. World Scientific.

[29] P. Wagner, K. Nagel, and D.E. Wolf. Realistic multi-lane traffic rules for cellular automata. Physica A, 234:687, 1997.

[30] D. Chowdhury, D.E. Wolf, and M. Schreckenberg. Particle hopping models for two-lane traffic with two kinds of vehicles: Effects of lane-changing rules. Physica A, 235(3-4):687, 1997.

[31] J. Krug and P.A. Ferrari. Phase transitions in driven diffusive systems with random rates. Journal of Physics A - Mathematical and General, 29:L465-L471, 1996.

[32] D.V. Ktitarev, D. Chowdhury, and D.E. Wolf. Stochastic traffic model with random deceleration probabilities: Queueing and power law gap distribution. Journal of Physics A, 30(8):L221, 1997.

[33] J. Treiterer and J.A. Myers. The hysteresis phenomenon in traffic flow. In D.J. Buckley, editor, Proc. 6th ISTT, page 13, Artarmon, New South Wales, 1974. A.H. \& A.W. Reed Pty Ltd.

[34] K. Nagel and M. Schreckenberg. A cellular automaton model for freeway traffic. J. Phys. I France, 2:2221, 1992.

[35] S. Krauss. Towards a unified view of microscopic traffic flow theories, 1997.

[36] M. Bando, K. Hasebe, K. Nakanishi, A. Nakayama, A. Shibatan, and Y. Sugiyama. Phenomenological study of dynamical model of traffic flow. J. Physique I France, 5(11), 1995. 


\begin{tabular}{|c|c|}
\hline $\begin{array}{l}\text { Schütt }[14]: \\
R \rightarrow L: v_{r}<v(\text { look-ahead distance }=9) \\
L \rightarrow R: v_{r} \geq v(\text { look-ahead distance }=15)\end{array}$ & {$\left[-v_{\max }+1, v_{\max }\right]$} \\
\hline $\begin{array}{l}\text { Rickert asym1 Master thesis }[20]: \\
R \rightarrow L: \operatorname{gap}_{r}<\min \left[v+1, v_{\max }\right] . O R \cdot \operatorname{gap}_{l} \leq 2 \min \left[v+1, v_{\max }\right] \\
L \rightarrow R: \operatorname{gap}_{r} \geq \min \left[v+1, v_{\max }\right] . A N D . g \operatorname{gap}_{l}>2 \min \left[v+1, v_{\max }\right]\end{array}$ & {$\left[-v_{\max }, \min \left(v+1, v_{\max }\right)\right]$} \\
\hline 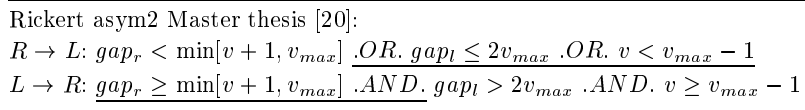 & {$\left[-v_{\max }, \min \left(v+1, v_{\max }\right)\right]$} \\
\hline $\begin{array}{l}\text { Latour } 1 \text { Master thesis }[23]: \\
R \rightarrow L: \operatorname{gap}_{r}<v \\
L \rightarrow R: \operatorname{gap}_{r} \geq v\end{array}$ & {$[0,0]$ (i.e. neighbor cell empty) } \\
\hline $\begin{array}{l}\text { Latour } 2 \text { Master thesis [23]: } \\
R \rightarrow L: \operatorname{gap}_{r}<f(v) . A N D . g_{a p}>\operatorname{gap}_{r}, f(v)=v, v+1, v+2, v_{\max } \\
L \rightarrow R: \operatorname{gap}_{r} \geq f(v) . \text {.OR. gap } \leq \text { gap } \\
\end{array}$ & a) $[0,0]$ b) $[-2,0]$ \\
\hline $\begin{array}{l}\text { Rickert et al Physica A [21]: } \\
R \rightarrow L: \operatorname{gap}_{r}<v+1 \\
L \rightarrow R: \operatorname{gap}_{r} \geq v+1\end{array}$ & {$\left[-\left(v_{\max }+1\right), v+1\right]$} \\
\hline 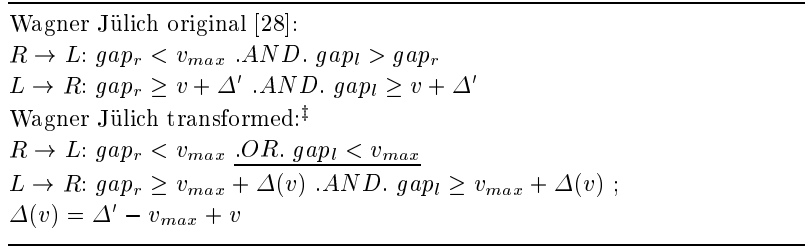 & $\begin{array}{l}{\left[-\left(v_{\text {back }}+1\right), 0\right]} \\
{\left[-\left(v_{\text {back }}+1\right), \min \left(\text { gap }+1, v_{\max }\right)\right]}\end{array}$ \\
\hline 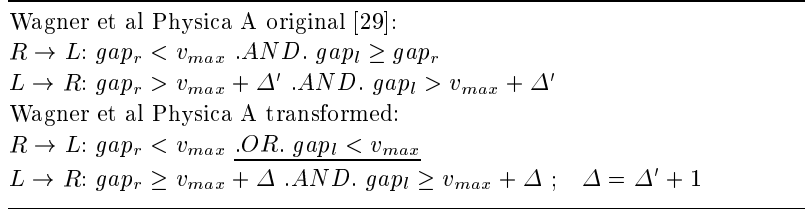 & $\begin{array}{l}{\left[-v_{\max }, 0\right]} \\
{\left[-v_{\max }, \min \left(g a p, v_{\max }\right)\right]}\end{array}$ \\
\hline $\begin{array}{l}\text { Chowdhury et al Physica A [30]: } \\
R \rightarrow L: \operatorname{gap}_{r}<v . O R . v_{d}>v_{d, r} \quad\left(\text { look-ahead distance } v_{\max }\right) \\
L \rightarrow R: \operatorname{gap}_{r} \geq v . A N D . v_{d} \leq v_{d, r} \quad\left(\text { look-ahead distance } v_{\max }\right)\end{array}$ & $\begin{array}{l}{\left[-v_{d, b a c k}, g a p\right]} \\
{\left[-v_{d, b a c k}, v\right]}\end{array}$ \\
\hline $\begin{array}{l}\text { This paper (velocity): } \\
R \rightarrow L: v_{r} \leq v . O R . v_{l} \leq v(\text { look-ahead distance }=16) \\
L \rightarrow R: v_{r}>v+\Delta . A N D . v_{l}>v+\Delta(\text { look-ahead distance }=16)\end{array}$ & {$\left[-v_{\max }, v\right]$} \\
\hline $\begin{array}{l}\text { This paper (gap): } \\
R \rightarrow L: \operatorname{gap}_{r}<v_{\max } . \text { OR. gapl }<v_{\max } \\
L \rightarrow R: \operatorname{gap}_{r} \geq v_{\max }+\Delta . A N D . \operatorname{gap}_{l} \geq v_{\max }+\Delta\end{array}$ & {$\left[-v_{\max }, v\right]$} \\
\hline
\end{tabular}

Table 1: Lane changing rules in the literature. The left column gives the "incentives to change lane" for the indicated lane change right to left $(R \rightarrow L)$ or left to right $(L \rightarrow R)$. The right colomn gives the security criterion, i.e. the sites on the target lane that need to be empty. Underlined parts need to be added to make the incentive to go right the logical negation of the incentive to go left. "look-ahead distance" is the distance to look ahead. $v_{b a c k}$ is the velocity of the next vehicle behind on the target lane. $v_{d}$ is the desired speed (i.e. may be smaller than $v_{\max }$ to denote a slower vehicle class. $v_{d, r}$ is the desired speed of the next vehicle ahead on the right lane. $v_{d, b a c k}$ is the desired speed of the next vehicle behind on the target lane. ${ }^{\ddagger}$ See appendix of this paper. 